\title{
Drugs targeting mGluR5 receptor offer 'fragile' hope for autism
}

A group of drugs that are already racing through clinical trials to treat fragile $\mathrm{X}$ syndrome, a rare inherited syndrome that causes both intellectual disability and autistic behaviors, may have even broader usage. Mounting evidence suggests that the drugs, which block a glutamate receptor in the brain called mGluR5, might also work in certain forms of autism that have no known cause. In recent weeks, studies have reported that mGluR5 drugs alleviate some symptoms in mouse models of autism and have found numerous genetic mutations in proteins downstream of mGluR5's signaling in people with the disorder.

"From the beginning, our hope was that insights into the pathophysiology of fragile $\mathrm{X}$ would apply to idiopathic autism," says Mark Bear, a neuroscientist at the Massachusetts Institute of Technology in Cambridge who reported in April that blocking mGluR5 reversed the signs of fragile $\mathrm{X}$ in a mouse model of the disease (Neuron 74, 49-56, 2012). "So we're thrilled."

In fragile $\mathrm{X}$ syndrome, mutations in a signaling molecule known as fragile $\mathrm{X}$ mental retardation protein (FMRP) prevent it from performing its normal role of damping mGluR5's activity, among other effects. The resulting constant activation of the receptor, Bear has shown, leads to excess protein production in neurons. This excess protein synthesis-although not fully understood-causes alterations in the structure and function of the neurons. Drugs that target mGluR5 work by putting the brakes back on the receptor and dialing down protein production. And companies see promise in this approach: Roche, Novartis and Seaside Therapeutics-a company founded by Bear-all have mGluR5 antagonists in clinical trials for fragile X.

Until recently, the idea that mutations in mGluR5-or proteins downstream from the receptor-could cause autism independently of fragile X was only a hunch. Now, the idea has become a theory with data to back it up.

In the 25 April issue of Science Translational Medicine (4, 131ra51, 2012), a team of scientists from the US National
Institute of Mental Health (NIMH) in Bethesda, Maryland and the New Yorkbased drug giant Pfizer reported that GRN529, an mGluR5 antagonist, decreased symptoms of autism in two different mouse models of the disease. The first type of mouse used in the study, an inbred strain, typically shows repetitive grooming-seen by scientists as symbolic of the repetitive behaviors of children with autism-and tend to vocalize and socialize less than other mice. When treated with GNR-529, the mice spent a quarter less time grooming themselves and showed some improvements in social interactions. The second group of mice tested

"Drugs targeting
mGluR5 could
have broader
applicability in
autism."
are known for repetitive jumping, also viewed as indicative of the repetitive behaviors seen in people with autism, and jumped less than a third as much after treatment with the mGluR5 antagonist.

But, since it's unknown whether either type of mouse has an mGluR5 mutation, the mechanism by which GRN-529 helps them remains unproven. The drug could have broad antianxiety effects, for example, that alleviate some symptoms of autism even if they're not targeting particular mutations in the mGluR5 pathway. "This means that we don't know yet, in humans, which patients would be helped by drugs that work through the mGluR5 pathway," says Jacqueline Crawley, head of the NIMH's Laboratory of Behavioral Neuroscience, who led the study.

Notably, recent sequencing studies on autistic children have also turned up mutations in a number of genes in the mGluR5 network. Publishing in Neuron (74, 285-299, 2012) on 26 April, geneticist Michael Wigler and his colleagues at the Cold Spring Harbor Laboratory in New York State analyzed the overlap between autism susceptibility genes and those already implicated as being misregulated in fragile $\mathrm{X}$ syndrome. They analyzed the overlap between autism susceptibility genes and those already implicated as being misregulated in

\begin{tabular}{|c|c|c|}
\hline \multicolumn{3}{|c|}{ Fragile promise: mGluR5 antagonists in the clinic for the treatment of fragile $\mathrm{X}$. } \\
\hline Company & Drug & Phase \\
\hline Novartis & AFQ056 & 3 \\
\hline Roche & R04917523 & 2 \\
\hline Seaside Therapeutics & STX107 & 2 \\
\hline
\end{tabular}

fragile X syndrome. Of 59 new autism genes they discovered, 14 were associated with FMRP, which is both the protein mutated in fragile $\mathrm{X}$ and the upstream regulator of the gene encoding mGluR5.

\section{Variation on a theme}

Raymond Kelleher, a neuroscientist at the Massachusetts General Hospital in Boston, took a more targeted look at the issue, inspecting the single-nucleotide variants in 18 mGluR5 pathway genes in 209 people with autism and 300 ethnically matched controls. His team reported in April that the idiopathic autism cases had higher burdens of mGluR5 variants. Three genes in the mGluR5 pathway that were previously implicated in autism associated with diseases such as fragile $\mathrm{X}$ and tuberous sclerosis-TSC1, TSC2 and SHANK3-were confirmed to be significantly different in the idiopathic autism population. They also found a fourth gene, never before implicated in autism, to be relevant. The gene, HOMER1, produces a protein that interacts in the cell with both the SHANK3 protein product and mGluR5 (PLoS One 7, e35003, 2012)

"This fits right in with our hypothesis that genetic variation in any members of this pathway could be overrepresented in autism," Kelleher says. "And if modulation of the mGluR5 pathway turns out to be successful in treating fragile $\mathrm{X}$, these sorts of results suggest that the treatment could have broader applicability in autism."

The next step for uncovering the role of mGluR5, researchers agree, is to test inhibitors of the receptor in mouse models of autism that contain genes known to be implicated in the human disease, rather than inbred mouse strains designed for the behavioral phenotype (see Nat. Med. 17, 1324, 2011). But whatever the outcome of those experiments, it's likely that mGluR5 drugs, once approved for fragile $\mathrm{X}$ syndrome, will be studied in human clinical trials for autism.

"Mice are not humans, and it would be a mistake to assume that an alteration in mouse behavior predicts human outcomes," says Bear. "But, that being said, right now we have an extremely strong rationale to test these drugs in humans and to cast a very wide net when we do."

Sarah C P Williams 